\title{
PSO Based Polynomial RST Controller Design and Sensitivity Analysis
}

\author{
Deepika $^{1}$, Dr. Shiv Narayan ${ }^{2}$ \\ M.E. Scholar, EED, PEC University of Technology, Chandigarh, India ${ }^{1}$ \\ Professor, EED, PEC University of Technology, Chandigarh, India ${ }^{2}$
}

\begin{abstract}
The objective of this paper is to implement the robust polynomial (RST) controller using particle swarm optimization technique and analyze the robustness properties of the closed loop system with the effect of the auxiliary poles on the output sensitivity function. This designing and analysis part is performed by taking design example from literature.
\end{abstract}

Keywords: PSO, Pole Placement, RST controller, Auxiliary poles, Sensitivity function.

\section{INTRODUCTION}

There are various types of the discrete controllers developed so far. One of the discrete polynomial controller is three part controller RST. One way of designing such controller is already developed by I.D. Landua[1][3][4]. Other way is an optimization algorithm. Also, there are various optimization techniques for the synthesis of RST controllers. In this paper, particle swarm optimization (PSO) is used for the designing purpose[2]. This algorithm is used to obtain the best and optimal values of controller coefficients by minimizing the integral absolute error of the output. This PSO method is used because of its simplicity and ease of implementation. It is faster as the number of iterations are lower than other popular techniques like genetic algorithm (GA). Till now in [2], the development of RST controller has already been done using PSO and it was also compared with GA.

But, in this paper, the new study is further done which is sensitivity analysis with auxiliary poles. This study is very important from robustness point of view, as the robust and good controller has always been demanded for the controlled process.

In this paper, firstly the robust polynomial RST controller has been designed using particle swarm optimization algorithm. Then, the robustness properties of the closed loop system is analyzed with varying auxiliary poles on its output sensitivity function. The auxiliary poles improve the robustness properties of the closed loop system.

The reciprocal of the output sensitivity function is called as the modulus margin which is one of the properties that is to be taken into account for verifying robustness of the system. The design example taken in this paper is dc drive described in later sections of the paper.

\section{PARTICLE SWARM OPTIMIZATION} (PSO)

PSO is one of a powerful optimization methods with high efficiency in comparison to other methods. It is a stochastic Evolutionary Computation technique based on the movement and intelligence of swarms. The PSO mechanism is initialized with a population of random solutions and searches for optima by updating generations
[2]. A swarm consists of $\mathrm{N}$ particles that are moving around in a D dimensional search space. Each particle keeps track of its coordinates in the space of the problem, which are associated with the best solution (best fitness) it has achieved so far. The best particle in the population is denoted by (gbest), while the best position that has been visited by the current particle is denoted by (pbest). The global best individual connects all members of the population to one another. That is, each particle is influenced by every best performance of any member in the entire population. The local best individual is seen as the ability for particles to remember past personal success. The particle swarm optimization concept involves, at each time step, changing the velocity of each particle towards its global best and local best locations.[2] The particles are manipulated according to the following equations of motion:

$$
\begin{aligned}
& v_{i d}^{k+1}=v_{i d}^{k}+c_{1} * r_{1} *\left(p_{i d}^{k}-x_{i d}^{k}\right)+c_{2} * r_{2} *\left(p_{g d}^{k}-x_{i d}^{k}\right) \\
& x_{i d}^{k+1}=x_{i d}^{k}+v_{i d}^{k}
\end{aligned}
$$

Where $\mathrm{v}$ is the velocity, $\mathrm{x}$ is the position, $\mathrm{p}_{\text {id }}$ and $\mathrm{g}_{\text {id }}$ are the pbest and gbest, $\mathrm{k}$ is iteration and $\mathrm{c}_{1}, \mathrm{c}_{2}$ are the cognition and social parameter. These parameters are variable or constant. Generally these values are ' 2 ' and $r_{1}, r_{2}$ are the random numbers in the range $(0,1)$.

\section{POLE PLACEMENT}

The pole placement strategy allows the design of a RST digital controller both for stable and unstable systems[1][3][4]:

1. It allows no restriction on the degrees of the polynomials A and B (1) of the discrete-time plant model (provided that they do not have common factors) [1].

2. No constraint on the time delay.

3. No constraint on the plant zeros (stable or unstable)

This method does not simplify the system zeros (this is why they can be unstable). The only problem is to take care of the possible common factors of A and B, which must be simplified before the computations are carried out. 
INTERNATIONAL JOURNAL OF INNOVATIVE RESEARCH IN ELECTRICAL, ELECTRONICS, INSTRUMENTATION AND CONTROL ENGINEERING Vol. 3, Issue 3, March 2015

\section{A. $\quad$ Structure of the RST Controller}

The structure of the closed loop system is given in Fig1.[4] The plant to be controlled is characterized by the pulse transfer function (irreducible):

$$
H\left(z^{-1}\right)=\frac{z^{-d} B\left(z^{-1}\right)}{A\left(z^{-1}\right)}
$$

in which $\mathrm{d}$ is the integer number of sampling periods contained in the time delay. Here, $\mathrm{q}^{-1}=\mathrm{z}^{-1}$ (shift operator). Here, the R,S,T polynomials denote the controller parameters[1][3].

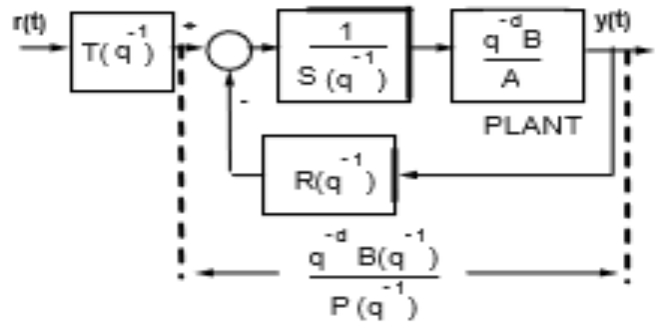

Fig 1 Pole placement with RST controller

The polynomials A,B of the plant is of the form:

$$
\begin{aligned}
& B\left(z^{-1}\right)=b_{1} z^{-1}+b_{2} z^{-2}+\ldots \ldots+b_{n} z^{-n} \\
& A\left(z^{-1}\right)=1+a_{1} z^{-1}+a_{2} z^{-2}+\ldots \ldots+a_{n^{\prime}} z^{-n^{\prime}}
\end{aligned}
$$

The polynomials R,S,T are given by:

$$
\begin{aligned}
& R\left(z^{-1}\right)=r_{0}+r_{1} z^{-1}+r_{2} z^{-2}+\ldots \ldots+r_{n r} z^{-n r} \\
& S\left(z^{-1}\right)=s_{0}+s_{1} z^{-1}+s_{2} z^{-2}+\ldots \ldots+s_{n s} z^{-n s} \\
& T\left(z^{-1}\right)=t_{0}+t_{1} z^{-1}+t_{2} z^{-2}+\ldots \ldots+t_{n t} z^{-n t}
\end{aligned}
$$

\section{B. Loop Shaping Of Sensitivity Functions with Pole} Placement

The sensitivity functions are very important for verifying the robustness properties of the closed loop system. These functions are shaped for the rejection of the disturbances and stability of the whole system[2][19]. If we take into account the output disturbance represented by $\mathrm{p}(\mathrm{t})$ then, the output sensitivity function is to be concerned. Its transfer function is given by:

$S_{y p}=\frac{A\left(z^{-1}\right) S\left(z^{-1}\right)}{P\left(z^{-1}\right)}$

Here the desired poles are given by the product of the dominant $\mathrm{P}_{\mathrm{D}}$ and auxiliary poles $\mathrm{P}_{\mathrm{F}}$. The auxiliary poles are known for improving the robustness performance of the closed loop system. The RST polynomials can be got from the bezout equation given by:

$$
A\left(z^{-1}\right) S\left(z^{-1}\right)+z^{-d} B\left(z^{-1}\right) R\left(z^{-1}\right)=P_{D}\left(z^{-1}\right) P_{F}\left(z^{-1}\right)
$$

But, in this paper, optimization technique has been formulated for finding the polynomials R,S,T of the controller.

In addition, the effect of the auxiliary poles is verified on the loop shaping of the sensitivity function. Larger or faster the auxiliary poles, lower is the maximum value of the output sensitivity function or modulus margin is improved.

\section{DESIGN EXAMPLE}

In this paper, the dc drive is taken as a design example[2]. The pulse transfer function is given by:

$$
H(z)=\frac{0.0005 z+0.0004}{z^{2}-1.431 z+0.4482}
$$

The polynomials R,S,T are given by:

$$
\begin{aligned}
& R\left(z^{-1}\right)=r_{0}+r_{1} z^{-1} \\
& S\left(z^{-1}\right)=s_{0}+s_{1} z^{-1}
\end{aligned}
$$

$T\left(z^{-1}\right)=1$

Aim is to design the RST controller such that[1][2][3]

Minimize $\mathbf{J}$ where

$J=\int_{0}^{\infty}|e(t)| d t$

Here $\mathbf{J}$ is an objective function i.e. integral of absolute error and error is the difference between the output and reference output.

\section{SIMULATION AND RESULTS}

\section{A. Design of RST Controller}

The polynomials R and S are coming out to be (16),(17) and (18) after applying PSO, taking swarm size as 20 as this gives the best fitness function value.

$\mathrm{T}=1$;

$R=0.01+0.02 z^{-1}$

$S=0.03+0.04 z^{-1}$

The step response for the closed loop system with polynomial controller is given in fig 2 . This shows that the system is stable as the steady state condition has been achieved in 8 seconds. Moreover, the peak overshoot is approximately $10 \%$ which is quiet less. Hence, the PSO algorithm gives best RST controller design. 
INTERNATIONAL JOURNAL OF INNOVATIVE RESEARCH IN ELECTRICAL, ELECTRONICS, INSTRUMENTATION AND CONTROL ENGINEERING Vol. 3, Issue 3, March 2015

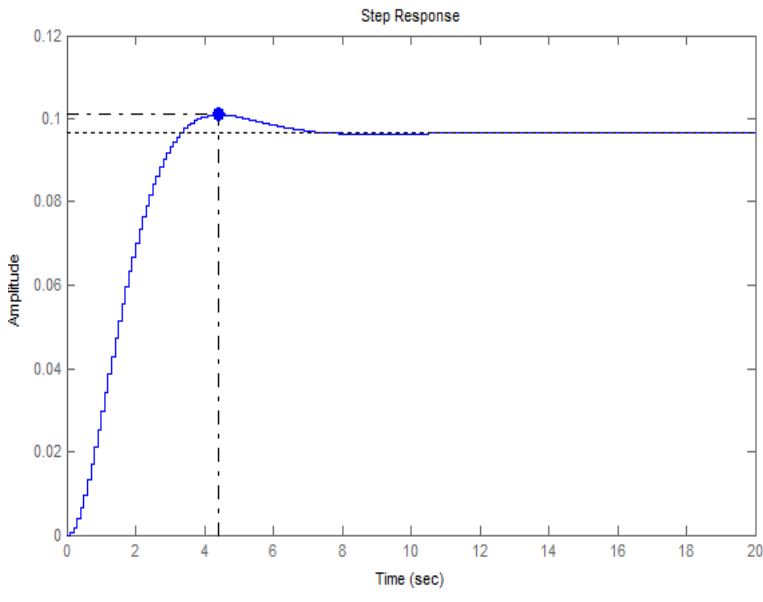

Fig 2. Step response for the closed loop system

The time performance characteristics for the closed loop system is given in table I.

TABLE I: PERFORMANCE SPECIFICATIONS

\begin{tabular}{|c|l|}
\hline Characteristics & Values \\
\hline Rise time & 2.1 seconds \\
\hline Settling time & 6 seconds \\
\hline Overshoot & $4.5 \%$ \\
\hline Peak & 0.1 \\
\hline Peak time & 4.4 second \\
\hline
\end{tabular}

$B$.

Analysis of the Closed Loop System

After further investigation, it has been found that by introducing larger auxiliary poles, the peak values of output sensitivity functions are decreasing. This can be easily visualized from the fig3. This shows that the modulus margin $(\Delta M>-6 \mathrm{db})[1][20]$ is increasing and therefore the system is becoming robustly stable. The auxiliary poles are taken as:

$P_{F 1}=1-0.1 z^{-1}$
$P_{F 2}=1-0.2 z^{-1}$
$P_{F 3}=1-0.3 z^{-1}$
$P_{F 4}=1-0.4 z^{-1}$

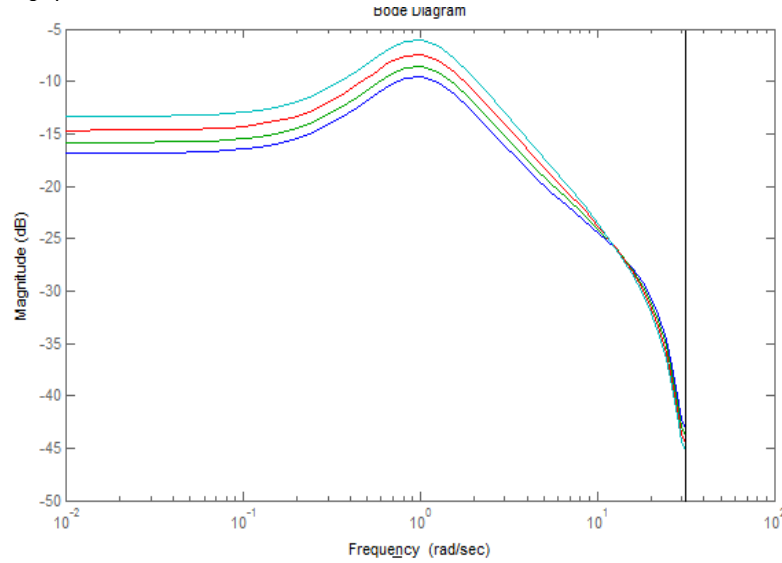

Fig 3. Bode plot for the output sensitivity functions with the variation in auxiliary poles

\section{CONCLUSION}

In this paper, firstly the RST polynomial controller has been formulated for the dc drive using particle swarm optimization algorithm by taking integral absolute error as an objective function. Then the analysis of the robustness properties of the closed loop system is done by varying auxiliary poles. This is verified by loop shaping of the output sensitivity function.

\section{ACKNOWLEDGMENT}

Thanks to my honorific guide Dr. Shiv Narayan, Professor, Department of Electrical Engineering, PEC University of technology, formerly Punjab Engineering College, Chandigarh with deepest gratitude, for giving the opportunities to perform research work under his able guidance.

\section{REFERENCES}

[1]. Ioan D. Landau and Gianluca Zito "'DigitalControl Systems”,1938

[2] .Riadh Madiouni, Soufiene Bouallegue, Josph Haggege and Patrick Siarry,' Particle Swarm Optimization-Based Design of Polynomial RST Controller', $10^{\text {th }}$ International Multi- Conference on Systems, Signals \& Device(SSD) Hammamet Tunisla, 2013.

[3]. Milan S. Matijević, Ranko Sredojevi and Vladimir M. Stojanovi," Robust RST Controller Design by Convex Optimization", ELECTRONICS, VOL. 15, NO. 1, JUNE 2011.

[4] .I. Landau, "The $R-S-T$ digital controller design and applications",Control Eng. Practice, Vol.6, 155-165, 1998.

[5]. K. Astrom and B. Wittenmark, Computer Controlled Systems. Theory and Design, Englewood Ciffs, NJ: Prentice-Hall, 1996.

[6]. M.S. Matijevic,Development of new structures of digitally controls electromotor drives and industrial processes, $\mathrm{PhD}$ thesis, Faculty of Mechanical Engineering, Kragujevac, 2001. (in Serbian)

[7]. E. Ostertag,. Godoy, RST-Controller Design for Sinewave References by Means of an Auxiliary Diophantine Equation, 44th IEEE Conference on Decision and Control, 2005 and 2005 European Control Conference. CDC-ECC '05,12-15 Dec. 2005.

[8]. H. Gharsallaoui, M. Ayadi, M. Benrejeb, P. Borne, Flatness-based control and conventional RST polynomial control of a thermal process, Int. J. of Computers, Communications \& Control, ISSN 1841-9836, E-ISSN 1841-9844, Vol. IV (2009), No. 1, pp. 41-56

[9]. Ya.Z. Tsypkin and U. Holmberg,"Robust stochastic control using the internal model principle and internal model control'Int. J. Control, vol. 61, No4, P 809-822, 1995.

[10]. J. Langer, I.D. Landau, "Combined pole placement/sensitivity function shaping method using convex optimization criteria",Automatica , 35, P 1111-1120, 1999.

[11]. S.P. Boyd and L. Vandenberghe, Convex optimization, Cambridge University Press, 2004

[12]. S.-P. Wu, S. Boyd, and L. Vandenberghe, "FIR filter design via semidefinite programming and spectral factorization,"Proc.IEEE Conf. on Decision and Control, 1996, pp. 271-276.

[13]. S. Bouallegue,J.Haggege,"A new method for tuning PID type fuzzy controllers using PSO". Chapter 6, pp.139-62, in fuzzy controllerrecent advances in theory and applications book,Croatia, 2012.

[14]. S. Bouallegue,J.Haggege, ” Structured Loop shaping Hœ Controller design using particle swarm optimization", proceedings of the 2010 IEEE, 2010.

[15]. ]. S. Bouallegue,J.Haggege,"structured mixed sensitivity $H \infty$ design using particle swarm optimization", proceedings of the $7^{\text {th }}$ IEEE ,SSD, 2010, Amman.

[16]. S.N. Vukosavic and M.R. Stojic, „Suppression of torsional oscillations in a high-performance speed servo drive",IEEE Trans. on Industrial Electronics, vol. 45, No1, 108-117, 1998.

[17]. .C.W. Ramos and P.L.D. Peres. A less conservative LMI condition for the robust stability of discrete-time uncertain systems.Systems \& Control Letters, 43:371-378,2001.

[18]. Kwakernaak H. Robust control and Hinf optimization - a tutorial Automatica, vol.29, pp.255-273,1993.

[19]. Sung H.K., Hara S. Properties of sensitivity and complementary sensitivity functions in single-input, single-output digital systems, Int. J. of Cont., vol. 48, n6, pp. 2429- 2439,1988.

[20]. Zhu K. Essentials of robust control, Prentice Hall, N.J., U.S.A,1998. 